\title{
Multi Energy Computed Tomography Imaging Optimization
}

\author{
Raziye Kubra Kumrular ${ }^{a}$, and Thomas Blumensath ${ }^{a}$ \\ ${ }^{a}$ Institute of Sound and Vibration Research, University of Southampton, Southampton, UK
}

\begin{abstract}
X-Ray Computed Tomography (CT) is a powerful imaging method because it can map spatial $X$ ray attenuation properties of an object. Lab-based X-ray sources generate photons of varying energies (the source spectrum), while laboratory-based X-ray detectors generally cannot resolve $X$-ray energies. These detectors measure the total amount of energy. This method is imperfect for the quantitative characterization of materials, as both material density and material composition will change overall $X$ ray transmission. Therefore, for material decomposition, an accurate model of the source spectrum and the detector's spectral response is needed in order to estimate absorption spectra when using non-energy resolving detectors. We develop computational methods that allow us to use a standard lab-based X-ray CT system to resolve the full range of possible materials using a multi-energy $X$-ray source and an energy integrating detector. We suggested achieving this by using a multi-energy scan approach coupled with the optimization of a more accurate $X$-ray absorption model. To overcome the ill-conditioning, we implement and study deep learning methods to constrain the inverse problem. We generated objects that have two chemical elements using NIST data at energies between $20 \mathrm{keV}$ and $200 \mathrm{keV}$. To learn a low dimensional model of absorption spectra, we used different auto-encoder models. We tested the method on a simplified 10-pixel problem with spectra quantised into 20 energy levels and generated one deep learning model to estimate material densities from the recovered absorption spectra. Our deep learning model was able to reliably predict one material of the two elements in the generated object.
\end{abstract}

Keywords - lab-based X-ray CT, material decomposition, deep learning, de-noising autoencoder

\section{SUMMARY}

We proposed the optimization of a more generic Xray tomography model. By imaging an object with a range of source spectra, we showed that spectral images could nevertheless be obtained, even if we have energy blind detectors. For observations to be integrated over energy levels, we tried to solve the problem by jointly estimating the X-ray measurement and X-ray absorption spectrum under the constraint. The research includes using DL (deep-learning) to learn a low-dimensional model of absorption spectra, using DL constraint to recover absorption spectra from X-ray measurements, and using DL to decompose absorption spectra estimates into material density estimates.

\section{DESCRIPTION OF Purpose}

The contrast in X-ray imaging is determined from the interaction between matter and X-ray photons. When Xray photons pass through an object, two possible events occur penetration and scattering. X-ray photons can penetrate without interacting with matter, which not gives information about the matter. However, when X-ray photons interact with the material can attenuate. Attenuation occurs by different scattering interactions and depends on the material and X-ray energy. Also, materials that have similar attenuation properties may appear almost identical in conventional X-ray CT [1], [2]. Although the source spectrum generates the poly-energetic X-ray spectrum, detectors in conventional X-ray imaging measure the total amount of energy because of integration over the energy spectrum. Because the interactions involving CT measurements are energy-dependent, energy integration leads to a nonlinear situation in how the physical properties of matter are measured and calculated [3]. This situation, therefore, makes the differentiation and quantification of materials extremely challenging. Spectral X-ray imaging takes advantage of the energy dependency of the linear attenuation coefficients (LAC) to yield energy-sensitive images. However, spectral CT imaging has some limitations, such as essential in instrumentation expensive energy-resolving detectors and a simplified X-ray absorption model that works for a group of materials with low atomic numbers $(Z \leq 15)$. Also, most studies with energy-solving detectors in the literature use eight energy bins, which are far from the ideal monochromatic energy assumption [4].

Estimating the material composition and densities of objects using conventional lab-based CT systems is currently not possible. This work will focus on the enhancement of accurate models (like X-ray absorption model, source spectrum, and ability to estimate absorption spectra when using non-energy resolving detectors) for the estimations of material. We aimed to develop computational methods that allow us to use a standard lab-based X-ray CT system to resolve the full range of possible materials using a multi-energy $\mathrm{X}$-ray source and an energy integrating detector. We aimed to achieve this goal by using a multi-energy scan approach coupled with the optimization of a more accurate X-ray absorption 
model. To overcome the ill-conditioning in this model, we suggested the use of several deep learning methods to constrain the inverse problem.

We can explain our aim with three hypotheses:

- Firstly, to use deep learning models to learn a low dimensional model of absorption spectra.

- Secondly, to recover absorption spectra from X-ray transmission measurements by constraining with the deep learning method.

- Lastly, to use deep learning to decompose spectral absorption spectra estimates into material density estimates.

\section{METHODS}

Estimating the material composition and densities of objects is required an accurate model of X-ray absorption spectra of the scanned object. We use a de-noising autoencoder to learn a low dimensional subspace to approximate $\mathrm{x}$-ray absorption spectra in this research. These models can be understood as an encoder and decoder. The encoder produces a code with few parameters, which then fully specifies the absorption spectrum using the decoder.

If we train a de-noising autoencoder to project onto noisy absorption spectra, then the closest point on the low dimensional manifold of absorption spectra can be found for any spectral estimate by mapping it through the autoencoder. When estimating spectra, this additional constraint can be included in the estimation of $\boldsymbol{x}(\boldsymbol{e})$ by mapping the absorption spectra estimate in each pixel through the trained autoencoder after each update. These estimated absorption spectra can be used in estimating material decomposition.

\section{A. De-noising Autoencoder for the Estimation of X-Ray} Absorption Spectra

To learn a low dimensional model of absorption spectra, simulated X-ray absorption spectra have been generated. When generating the data-set, the penetrating ability of the X-ray with the matter has been used. The linear attenuation coefficient of materials can be obtained by multiplying MAC (Mass attenuation coefficient) with mass density. Data from the National Institute of Standards and Technology (NIST) [5] have been used to obtain the MAC values and densities of chemical elements. The LAC $(\mu)$ values at energies between 20 and $200 \mathrm{keV}$ have been equally divided into 20 energy ranges, and 20 LAC values have been created for each element. Two different arbitrary scalar factors which are changing 0-1 have been used to combine two arbitrary LAC series. In this way, combined LACs that represent the X-ray penetrating ability for compound material has been created. Thus, simulated data-set has been generated for 20000 materials that have 20 LACs. Gaussian noise has been added to this data set to generate a noisy data set. A convolutional neural network $(\mathrm{CNN})$ and three different fully connected neural networks (FCNN) have been generated as an autoencoder. These networks have been used to denoise a noisy Gaussian spectrum.

\section{B. Recovery of X-Ray Absorption Spectra from X-Ray Measurements}

To recover the X-ray absorption spectrum $\boldsymbol{x}(\boldsymbol{e})$ from the measurement $y_{j, k}$ with the given estimated X-ray source spectrum $I_{k, e}$, de-noising autoencoder models have been used as a constraint in the optimization. Experiments in this section are carried out assumed Xray source spectrum is known. Also, Experiments have been done with random source spectra initiation, and 3-6 source spectra have been used. For observations that are integrated over energy levels, the problem has been tried to solve by jointly estimating the X-ray measurement $y_{j, k}$, and X-ray absorption spectrum $\boldsymbol{x}(\boldsymbol{e})$, under the constraint.

\section{Estimation of Material Densities from Absorption Spectra}

One deep learning architecture has been generated for material recovery. Input variables are X-ray absorption spectra, while output is a vector of 92 elements, which each of them represents the proportion of the chemical elements present in the object. These chemical elements have been arranged by atomic number, from hydrogen to uranium. With a neural network regression model, the proportion for the presence of each of the 92 chemical elements in the object has been calculated.

\section{Results}

\section{A. Performance of Denoising}

Generated simulated training data have been randomly divided into 3, training, validation, and test for the denoising process. In training, Gaussian noisy X-ray spectra have been used as an input, and noise-free X-ray spectra have been used as an output. After that, generated architectures have been trained with the training set. Finally, selected random test data have been used to test the denoising performance of the architectures. To make the comparison of denoising performance for generated three FCNN structures and $\mathrm{CNN}$, selected test data is shown in Fig1. The gap between the predicted $\mathrm{X}$ ray absorption spectrum by FCNN-1 and FCNN-3 and the actual X-ray absorption spectrum is relatively large than FCNN-2. Also, the gap between the predicted Xray absorption spectrum by FCNN-2 the actual X-ray absorption spectrum is relatively large than the predicted $\mathrm{X}$-ray absorption spectrum by $\mathrm{CNN}$. 



Fig. 1. Performance of $\mathrm{x}$-ray absorption spectrum denoising with different autoencoder architectures

\section{B. Performance in Recovering Spectra}

First of all, the number of source spectrum (3-6 source spectrum) has been defined. After that, a simplified 10pixel with spectra quantized into 20 energy levels has been defined, and 20 measurements have been got from these pixels. Two denoising autoencoder models have been used to project onto the low-dimensional data manifold in the optimization. These autoencoder models are FCNN-2 and CNN, have been mentioned in section IV.A. Generated deep learning architectures have been used as a constraint for recovered spectrum estimation in every ten iterations. The cost has been expected to be lower after every iteration as the recovered spectrum comes closer to the true spectrum. A set of randomly selected data has been plotted to visualize the performance of recovering spectra from X-ray measurement, as shown in fig2. It has been seen that the performance of $\mathrm{CCN}$ and FCNN-2 for recovery from measurement is so similar also, still have error between the real one and recovered. In this step, the noise ratio has been used as an evaluation metric to evaluate the performance recovery method. The noise ratio of the FCNN-2 used as a constraint in optimization processes is lower than the noise ratio of CNN also; training run time is the opposite. With the number of source spectra increasing, the noise ratios of decrease. However, the trade-off is the massively increased run time of code.

\section{Performance of Materials Recovering}

Firstly, generated CNN architecture has been used for material recovery from the simulated X-ray absorption spectrum mentioned in section III.A. After that, deep learning architecture trained with simulated data has been used for material recovery from the recovered $\mathrm{X}$ ray absorption spectrum in section III.B. The X-ray absorption spectrums have been defined as the inputs of the network, while the proportion of elements in the generated object have been defined as the output of the network.
When the generated object consisted of lower atomic number elements (like Lithium), architecture has been could not accurately predict the ratio of them. The generated object consists of Lithium and Caesium, but the architecture has estimated Lithium and Caesium, as well as other elements such as Beryllium and Helium with a minor proportion, as shown left in fig3. The generated network could not accurately predict two materials when used to recover the material from the recovered X-ray absorption spectrum from X-ray measurements. The one material in the generated object has been predicted. Even if the generated object consists of sodium and polonium, the network only has predicted sodium with a low ratio than the actual ratio and could not predict polonium. In addition, the network has estimated Aluminium with a high value and other elements with lower values. However, the object does not contain these elements, as shown right in fig3.

\section{CONCLUSION}

This paper proposes a systematic and complete process to get the components of an object from measurements on the X-ray detectors using the different deep learning models. In the first part, generated auto-encoders using FCNN and CNN have been used for denoising from the simulated X-ray absorption spectrum data-set. Especially three different FCNN network architectures were searched to test denoising performance, and FCNN2 showed the best result when compared to the other FCNN test structures. Also, the performance of FCNN-2 was compared to the $\mathrm{CNN}$ for denoising. A small error gap between the denoised X-ray absorption spectrum by these two networks (FCNN-2 and $\mathrm{CNN}$ ) and the true Xray absorption spectrum have been observed. However, $\mathrm{CNN}$ has been outperformed FCNN-2 in denoising.

In the second part, the mathematical optimization method is used to recover the spectra from measurements. One of the constraints in the mathematical optimization method has been defined as the generated denoising neural networks. Experiments were carried out 

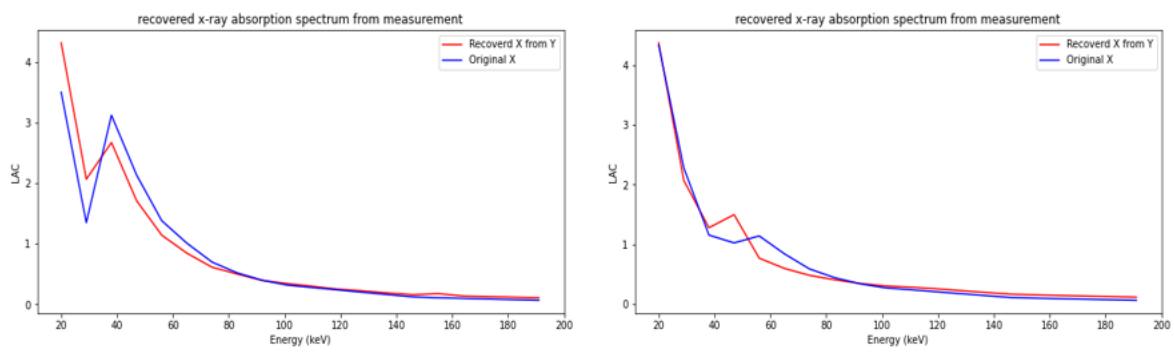

Fig. 2. The plot of recovered spectrum and real spectrum. left: CNN used as a constraint, right: FCNN-2 used as a constraint.

TABLE I

SPECTRUM RECOVERY EXPERIMENT RESULTS

\begin{tabular}{||ccccc||}
\hline Test structure & Test number & Source number & Noise ratio & Training run-time/s \\
\hline \hline CNN & Average & 3 & 0.096 & 157.845 \\
\hline FCNN-2 & Average & 3 & 0.081 & 192.121 \\
\hline CNN & Average & 6 & 0.089 & 780.932 \\
\hline FCNN-2 & Average & 6 & 0.069 & 814.014 \\
\hline
\end{tabular}
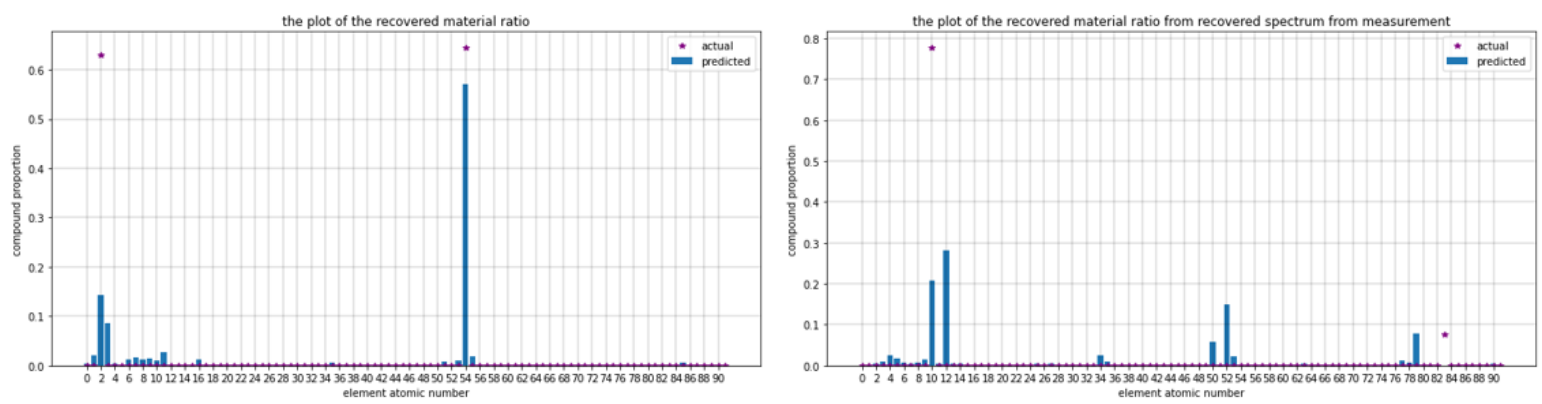

Fig. 3. The plot of recovered materials proportion. The numbers on the x-axis represent the atomic number, and the numbers on the $y$-axis stand for the component proportion. The $\mathrm{x}$-axis is shown two by two and starts from 0 . left: material recovery of the simulated $\mathrm{x}$-ray absorption spectrum, right: material recovery of recovered $\mathrm{x}$-ray absorption spectrum from $\mathrm{x}$-ray measurement

to decide which neural network was more suitable to work as one constraint in this mathematical optimization. In these experiments, FCNN-2 were outperformed the $\mathrm{CNN}$ in this optimization with a good noise ratio despite the run-time.

In the last part, another $\mathrm{CNN}$ network structure is generated for material recovery. This network is experimented with for material recovery from simulated X-ray absorption spectra. The predictive ability of the network is not good if the generated object consists of low atomic number material. The network for these objects predicted much more material than existed in addition to the actual two materials. After this experiment, the generated network for material estimation was used to recover materials from the recovered spectrum from measurement. The network has predicted just one material with a different ratio than the actual ratio and the other materials, which don't exist in the generated object.

All the algorithms implemented in this paper are done in Python coding language, which can be used for further development. All deep learning architectures have been created using Keras [6].

\section{REFERENCES}

[1] D. Mery, "Computer vision for x-ray testing," Switzerland: Springer International Publishing, vol. 10, pp. 978-3, 2015.

[2] R. Bhayana, A. Parakh, and A. Kambadakone, "Material decomposition with dual-and multi-energy computed tomography," MRS Communications, vol. 10, no. 4, pp. 558-565, 2020.

[3] S. J. Nik, "Optimising the benefits of spectral x-ray imaging in material decomposition," 2013.

[4] M. Busi, "Enhanced security screening using spectral x-ray imaging," 2019.

[5] N. I. of Standards and Technology, "X-ray mass attenuation coefficients," accessed Jun 03, 2021. [Online]. Available: https://www.nist.gov/pml/x-ray-mass-attenuation-coefficients

[6] Keras, "Keras: Deep learning for humans," accessed Jun. 07, 2021. [Online]. Available: https://github.com/keras-team/keras 